\title{
Clinical and surgical algorithm for managing iatrogenic bile duct injuries during laparoscopic cholecystectomy: A multicenter study
}

\author{
COSMIN MOLDOVAN $^{1,2}$, DANIEL COCHIOR ${ }^{1,3,4}$, GABRIEL GORECKI ${ }^{5}$, \\ ELENA RUSU $^{1}$ and FLORIN-DAN UNGUREANU ${ }^{1}$
}

\author{
${ }^{1}$ Faculty of Medicine, 'Titu Maiorescu' University, 040441 Bucharest; ${ }^{2}$ General Surgery Ward, \\ 'Witting' Clinical Hospital, 010243 Bucharest; ${ }^{3}$ General Surgery, 'Sanador' Clinical Hospital, \\ 010991 Bucharest; ${ }^{4}$ General Surgery, 'Monza' Clinical Hospital, 021967 Bucharest; \\ ${ }^{5}$ Medicine Doctoral School, 'Titu Maiorescu' University, 040317 Bucharest, Romania
}

Received July 12, 2021; Accepted August 11, 2021

DOI: $10.3892 / \mathrm{etm} .2021 .10821$

\begin{abstract}
The present study was a multicenter, analytical, nonrandomized research on 108 cases of intraoperative vascular and bile duct lesions during laparoscopic cholecystectomies. We selected these cases from 16,559 cholecystectomies performed entirely laparoscopically or debuted laparoscopically and converted to an open approach. The study included two surgical centers labeled as primary, with extensive experience in hepato-biliary reconstructive surgery, and four other centers labeled as secondary that referred cases to the previous two. Our study analyzed several key parameters such as the percentage of iatrogenic lesions recorded, the variability of the main biliary pathway and conformation as well as its relationship to the adjacent critical anatomical landmarks, the anatomical and physiopathological characteristics of pathology requiring surgical intervention, factors related to laparoscopic surgical technique, the surgical technique used to repair the recorded lesions, the duration of survivability and the rate of the occurring complications. Based on the analysis of these parameters, we developed a descriptive algorithm with visual representation relying on several decisional points to guide the surgeons in choosing the optimal treatment method so that patients will benefit from a favorable clinical path.
\end{abstract}

\section{Introduction}

The history of the treatment of vesicular lithiasis pathology has undergone one of the fastest medical technology developments in all surgical disciplines, experiencing the fastest transition from traditional to modern techniques. In 1985, Erich Mühe,

Correspondence to: Professor Daniel Cochior, Faculty of Medicine, 'Titu Maiorescu' University, 22 Dâmbovnicului Street, 040441 Bucharest, Romania

E-mail: cochiordaniel@gmail.com

Key words: iatrogenic, bile duct injury, laparoscopic cholecystectomy a radiologist by profession, performed the first endoscopic cholecystectomy using a tube-shaped instrument (1). Only four years later, following the Mühe breakthrough, the US reached a 90\% rate of laparoscopically performed cholecystectomy (2).

Despite the constant and consistent technological advances, iatrogenic lesions during laparoscopic cholecystectomies have not decreased. Moreover, the lesion profile has acquired increasingly severe aspects with long-term postoperative results confirming the significant impact of this pathology. During the laparoscopic cholecystectomy (LC) of lesions directly diagnosed intraoperatively and repaired in the same intervention by the surgeon, only $17 \%$ have a favorable path (3). Recent research shows a case study of 27 patients with significant bile duct injuries, in which the primary repair was conducted by the same team, with a recorded failure rate of $95 \%$ (4), a worrying percentage rate. In addition, in a retrospective analysis of 151 cases of malpractice following hepatobiliary laparoscopic surgery, researchers suggest a $42 \%$ rate for main biliary pathway (MBP) lesions $(5,6)$. Therefore we propose a guide for managing and recognizing different cases of iatrogenic lesions during laparoscopic cholecystectomies. Such a guide for direct intraoperative detection or early postoperative detection of iatrogenic lesions can constitute real support for any surgical department that addresses such a pathology.

\section{Patients and methods}

Our study is a multicenter, analytical, controlled research based on a 12-year retrospective analysis between January 2008 to December 2020. We selected 108 patients with various intraoperative iatrogenic lesions on the extrahepatic bile ducts with or without associated major vascular injuries. The selected patients were enrolled from 16,559 surgeries performed entirely laparoscopically or laparoscopically debuted and converted to the classical approach for benign vesicular pathology during the abovementioned period. The research took place at two clinics considered primary centers (CF1 Witting Clinical Hospital and CF2 Clinical Hospital in Bucharest, Romania) that received patients from four other centers in the province, considered secondary in this study. 
Classification of primary and secondary centers is based on the level of hepato-biliary reconstructive surgical experience.

Cases of iatrogenic lesions determined in the primary clinics and those transferred for complete diagnosis (etiological and staging) and definitive surgical repair in secondary centers were recorded. For this batch, the following parameters were considered: The percentage of iatrogenic lesions recorded, the variability of the main bile duct (MBD) in relationship with main adjacent important anatomical landmarks, the anatomical and physiopathological characteristics of the pathology that determined the respective surgical interventions, factors related to laparoscopic surgical technique, the surgical technique used to repair the recorded lesions, the overall survivability and rate of complications. Starting from the previously mentioned parameters, we created a study to identify the profiles and precise intraoperative situations that present the maximum risk of iatrogenic lesions. We also aimed to quantify the actual impact of these iatrogenic lesions based on the analysis of hospitalization costs, the number of required reinterventions, the period of admission, and clinical evolution.

Our research study resulted in a surgical conduct guide based on a decision-tree algorithm. We believe that we created a guide easy to use both intraoperatively and in the immediate postoperative period that can serve as a model for solving issues in regards to these iatrogenic lesions. Our guide is also a model applicable in secondary surgical services that do not benefit from the immediate help of reference centers in corrective surgery of iatrogenic lesions of the hepato-biliary tree.

For the present study aim, a new internal classification method was adopted, obtained by re-organizing the classical systems for reporting bile duct lesions, focusing more on describing lesion severity and less on full details of the lesions. Thus, we categorized the patients into three lesion groups, from Group A (the simplest) to Group C (the most severe). In Group A, we included bile losses from the cystic stump, lesions of the Lutschka duct, and damage to the right accessory hepatic duct (RAHD). Group B recorded cases of more advanced lesions of bile ducts, with no vascular damage, and Group C included complex vasculo-biliary combined lesions.

The equivalence between this internal case reporting system and traditional classification systems is documented in Table I. The Neuhaus and Strasberg-Bismuth classifications are not part of Group $\mathrm{C}$ because these systems cannot encode vascular lesions.

Primary statistical processing was performed through standard tools in Microsoft Excel 365, and advanced processing was performed with IBM SPSS ${ }^{\circledR}$ version 24 (IBM Corp.) and CDC Epi Info ${ }^{\mathrm{TM}}$ version 7.2.2.1 (https://www. cdc.gov/epiinfo/pc.html). Other statistical analysis methods consisted of tests that interpret continuous variables such as The Wilcoxon-Mann-Whitney (U-test) and the Kruskal-Wallis test (H-Test, a NOVA test), both of which are non-parametric. For assessing the accuracy of statistical processing, the P-value was set to 0.05 .

\section{Results}

The iatrogenic lesion rate incidence of laparoscopic cholecystectomy (LC) cases in our 12-year range study was different between the two types of clinics, ranging from $0.73 \%$ for primary clinics (80 cases out of 10,959 interventions practiced) to $0.5 \%$ (28 cases out of 5,600 interventions) for secondary clinics. The overall incidence rate of laparoscopic bile duct injuries (LBDI) in our study was $0.65 \%$.

The demographic data analysis revealed a ratio of 2.7:1 in favor of female patients with an average age of 47 years, with limits between 20 and 85 years. Most patients (83\%, 90 cases) came from urban areas. No specific analysis was used to identify the statistical relevance of these parameters.

Of the 108 cases in our study, 80 came from primary clinics and 28 from secondary clinics. In addition, from the total number of cases, $32(30.2 \%)$ lesions were diagnosed intraoperatively, and 76 lesions were established postoperatively $(69.8 \%)$. Of the 32 lesions directly identified intraoperatively, $40 \%$ (13 cases) were identified only by dedicated imaging, with the remaining 60\% (20 cases) directly observed. The imaging used consisted of intraoperative cholangiography (97\%) and endsocopic retrograde cholagiopancreatography (ERCP) in only $3 \%$ of cases. Cholangiography was used in its trans-choledochal variant ( 7 cases, 59\%), followed by the transcystic variant $(17 \%, 2$ cases) and in only 1 case in its trans-vesicular variant, which was a last resort solution. The diagnosis in the postoperative stage was based mainly on imaging investigations (92\%), including ERCP $(60 \%$, 46 cases), cholagio-MRI (20\%, 16 cases), trans-abdominal ultrasound (10\%, 8 cases) and cholangio-CT (2\%). In only $8 \%$ of the cases, the diagnosis was based on clinical and direct observation (signs of choleperitoneum and change in the peritoneal drainage aspect).

The vesicular pathology that established the laparoscopic approach surgical indication was $31.5 \%$ (34 cases) of acute diagnoses, such as acute lithiasic cholecystitis, acute lithiasic hydropiocholecystitis, vesicular hydrops, acute chronic cholecystitis (lithiasic or not). The remaining 74 cases $(68.5 \%)$ were represented by chronic diagnoses such as chronic cholecystitis (with or without lithiasis), and vesicular polyposis.

The conversion from laparoscopic intervention to open approach was recorded in 34 cases (31.5\%) and was based on several reasons, such as the need for staging the lesion and a correct and complete mapping through additional imaging tests or the attempt to repair the iatrogenic lesion during the same procedure.

An analysis of the distribution of the three groups revealed that $29 \%$ of the cases (31 patients) presented with the simplest lesions (Group A), 56\% of the cases (61 patients) present with Group B lesions, and $15 \%$ of the cases (16 patients) presented with the most severe lesions of Group C. As mentioned, our analysis consisted of many parameters, resulting in the following detailed description of the assigned groups (Table II).

The treatment methods used to repair the lesions, regardless of the lesion group, consisted of stenting (ST), simple peritoneal drainage (SD), simple suture (SS) of the bile lesion (laparoscopic or after conversion), bilio-digestive anastomosis (choledoco-jejunal anastomosis, choledoco-choledochal anastomosis, hepatico-jejunal anastomosis) and hepatic resection (HR). Without addressing the group in which they were practiced, their share was 56\% (61 cases) for hepato-jejunal anastomoses (HJA), simple sutures in 16\% (17 cases), stenting in $14 \%$ (15 cases), simple drainage in $6 \%$ (7 cases), liver resection (LR) in 5\% (5 cases) and $1 \%$ for choledochal-jejunal 
Table I. Correspondence between our classification system and the most cited classifications regarding vascular and bile duct lesions during cholecystectomies.

Lesional Group

\begin{tabular}{lcll}
\cline { 2 - 4 } Classification system & Group A & \multicolumn{1}{c}{ Group B } & \multicolumn{1}{c}{ Group C } \\
\hline Hannover & A1, A2 & B1, B2, C1, C2, C3, C4, D1, D2, D3, D4 & C2d, D2d, D3d, D3dpv, \\
& & & D3pv, D4c, D4d, D4dpv \\
Neuhaus & A1, A2 & B1, B2, C1, C2, D1, D2, E1, E2, E3 & n/a \\
Stewart-Way & I & II, III & IV \\
Strasberg-Bismuth & A & B, C, D, E1, E2, E3, E4, E5 & n/a \\
Siewert & I & II, IIIa, IIIb & IVa, IVb \\
\hline
\end{tabular}

n/a, not applicable.

Table II. Overall characteristics of the lesional groups in the study.

\begin{tabular}{|c|c|c|c|c|c|c|c|}
\hline \multirow[b]{3}{*}{ Parameter } & \multicolumn{6}{|c|}{ Group } & \multirow[b]{3}{*}{ P-value } \\
\hline & \multicolumn{2}{|c|}{ Group A } & \multicolumn{2}{|c|}{ Group B } & \multicolumn{2}{|c|}{ Group C } & \\
\hline & No. & $\%$ & No. & $\%$ & No. & $\%$ & \\
\hline Total & \multicolumn{2}{|c|}{$31(29 \%)$} & \multicolumn{2}{|c|}{$61(56 \%)$} & \multicolumn{2}{|c|}{$16(15 \%)$} & - \\
\hline Women & 19 & $61 \%$ & 47 & $77 \%$ & 10 & $62 \%$ & - \\
\hline Men & 12 & $39 \%$ & 14 & $23 \%$ & 6 & $38 \%$ & 0.328 \\
\hline Average age (years) & \multicolumn{2}{|c|}{46.2} & \multicolumn{2}{|c|}{44.9} & \multicolumn{2}{|c|}{54.5} & 0.130 \\
\hline Median hospitalization time (days) & \multicolumn{2}{|c|}{1.6} & \multicolumn{2}{|c|}{8.8} & \multicolumn{2}{|c|}{11.8} & $<0.001$ \\
\hline Median positive diagnosis time (days) & \multicolumn{2}{|c|}{2.5} & \multicolumn{2}{|c|}{3.0} & \multicolumn{2}{|c|}{2.5} & 0.796 \\
\hline Global rate of complications & 1 & $3.2 \%$ & 8 & $10.8 \%$ & 5 & $30 \%$ & 0.107 \\
\hline Late stenosis & 0 & 0 & 17 & $28 \%$ & 4 & $25 \%$ & 0.109 \\
\hline Mortality rates & 0 & 0 & 0 & 0 & 0 & 0 & 0.030 \\
\hline Medium costs (Lei) & \multicolumn{2}{|c|}{ 3075.69 Lei } & \multicolumn{2}{|c|}{ 11472.32 Lei } & \multicolumn{2}{|c|}{ 17131.59 Lei } & $<0.001$ \\
\hline \multirow[t]{2}{*}{ Medium costs (US/EUR) } & \multicolumn{2}{|c|}{$750 \mathrm{USD} /$} & \multicolumn{2}{|c|}{$2,860 \mathrm{USD} /$} & \multicolumn{2}{|c|}{$4,180 \mathrm{USD} /$} & \\
\hline & \multicolumn{2}{|c|}{630 EUR } & \multicolumn{2}{|c|}{ 2,400 EUR } & \multicolumn{2}{|c|}{ 3,506 EUR } & \\
\hline
\end{tabular}

anastomosis (CJA) and choledoco-choledochal anastomosis (CCA) (Fig. 1).

Regarding the attempted repair performed in the same surgical center that also determined LBDI, 28 patients were remitted from the secondary, less experienced in reconstructive surgery, clinics, to primary ones (26\% of all cases). However, the treatment methods chosen for LBDI repair varied between the primary and secondary centers; this was further correlated with a different clinical course for the patients. In the primary clinics, the following procedures were performed: HJA (46 cases), ST (15 cases), SD (7 cases) and SS (4 cases), while in the secondary clinics only HJA (15 cases) and SS (13 cases) were performed. Note that all cases of hepatic (HR) (5 patients) were fully practiced in the primary clinics (Fig. 1).

Regarding the treatment methods used in relation to the groups, it was noted that, for Group A, with the most benign lesions, SS was the most commonly practiced (15 cases) procedure, followed by ST (10 cases) and SD ( 6 cases). The rest of the treatment methods were not applied, as their complexity was not justified for such minor injuries. For Group B, with more advanced lesions, the distribution was different, with 50 cases of HJA, 5 cases of ST, 2 cases with SS and 1 case of CJA and CCA. For Lesion Group C, with the most advanced lesions, the surgical methods used were predominantly complex ones, with 11 cases of HJA and 5 cases of HR (Fig. 2).

The most common long-term complication in reconstructive surgery of extrahepatic bile ducts was, of course, stenosis. Thus our study remotely followed this complication through the following directions: the overall percentage of stenosis, stenosis rate correlated with lesional groups, surgical procedures chosen for repair and establishment of what types of surgical procedures triggered stenosis and their origin (performed in primary clinics or secondary ones). This study recorded stenosis according to Bismuth's classic 4-point classification system (7) which is still valid today despite being described in the days of open-approach only and even if in our study stenosis was the result of lesions caused by 


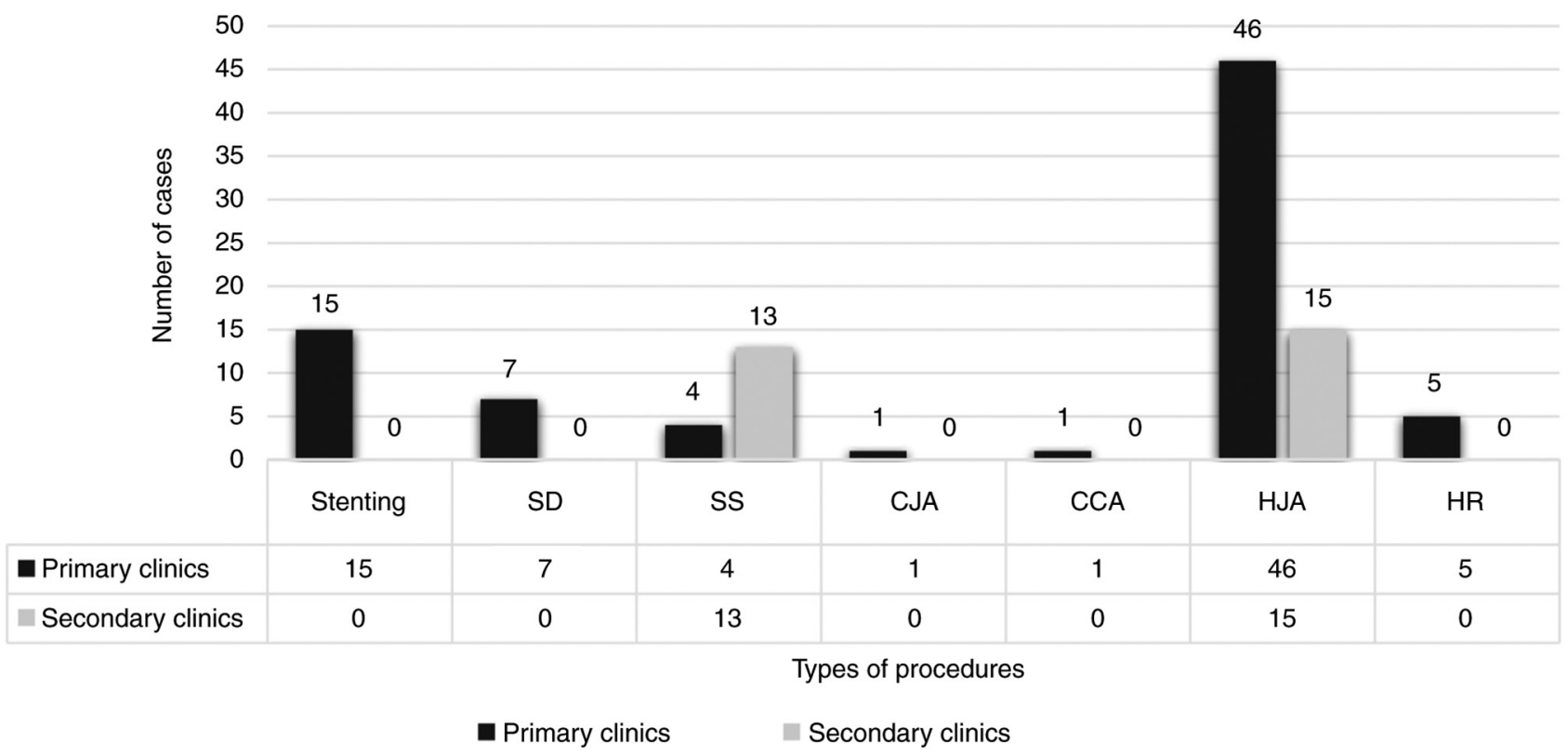

Figure 1. Comparative distribution of treatment methods between the primary and secondary clinics. SD, simple peritoneal drainage; SS, simple suture; CJA, choledoco-jejunal anastomosis; CCA, choledoco-choledochal anastomosis; HJA, hepatico-jejunal anastomosis; HR, hepatic resection.

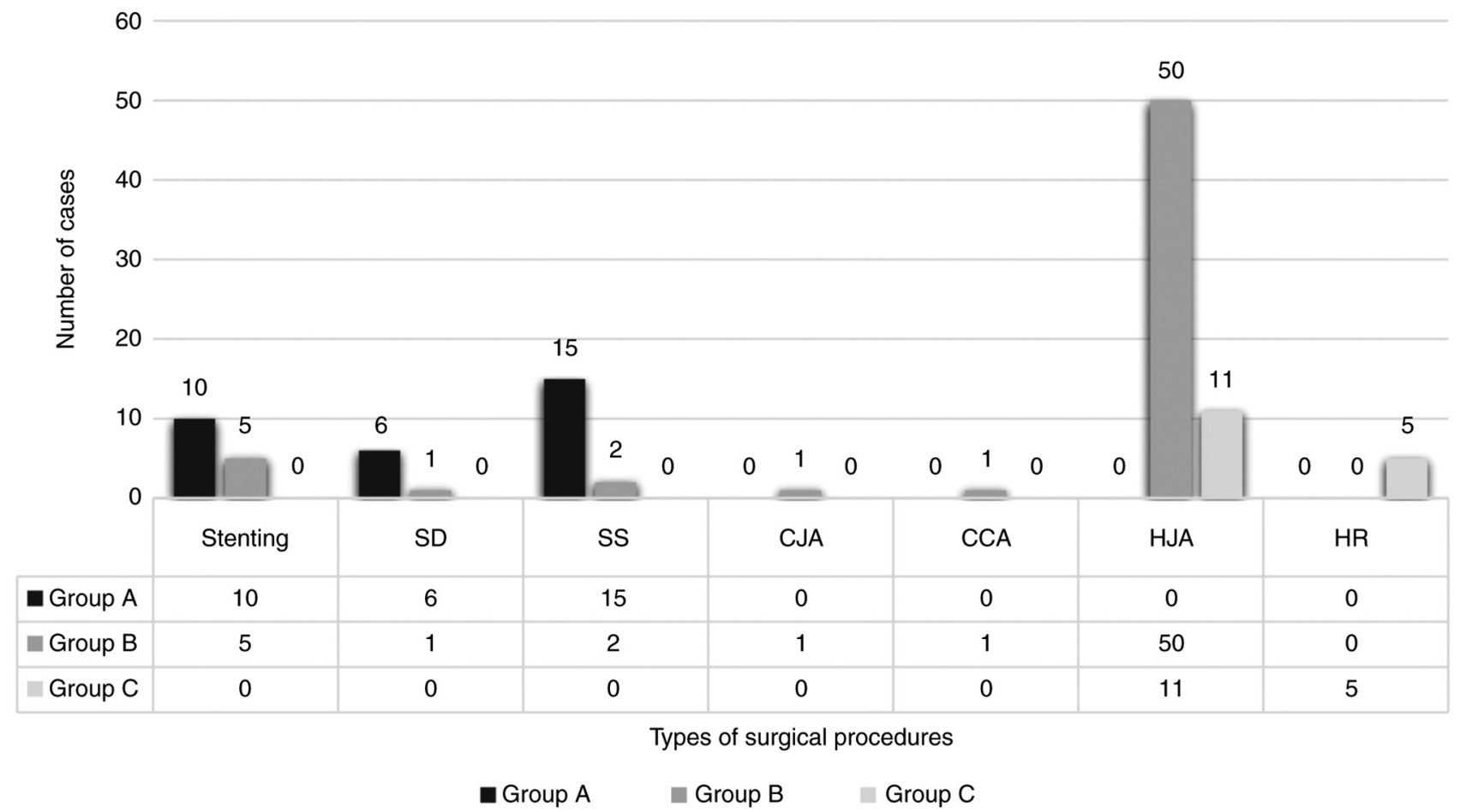

Figure 2. Comparative distribution of all treatment options in our study. SD, simple peritoneal drainage; SS, simple suture; CJA, choledoco-jejunal anastomosis; CCA, choledoco-choledochal anastomosis; HJA, hepatico-jejunal anastomosis; HR, hepatic resection.

laparoscopic surgery. The overall stenosis rate in the batch was 21 cases. These came exclusively from groups B and C $(81 \%$, 17 and 19\%, 4 cases, respectively). Group A did not cause any stenosis. Most stenosis developed from patients from secondary clinics $(62 \%, 13$ cases) compared to primary clinics that caused a stenosis rate of $38 \%$ (8 cases). Disregarding the centers where the interventions were performed, the analysis of surgical procedures chosen for LBDI repair that determined late-term stenosis revealed that CCA was credited with $100 \%$ stenosis rate followed by HJA with $26 \%$ and the last being SS, with $24 \%$. The procedure that did not cause any stenosis was CJA. Suppose we factor in the type of center where those surgical procedures were performed. In that case, we note that for HJA the highest number of cases with stenosis came from secondary clinics (10 case), compared to only 6 HJA performed in primary centers. The same situation was evident for SS in which 3 cases in secondary centers determined stenosis and only 1 case was present in primary centers (Fig. 3). 


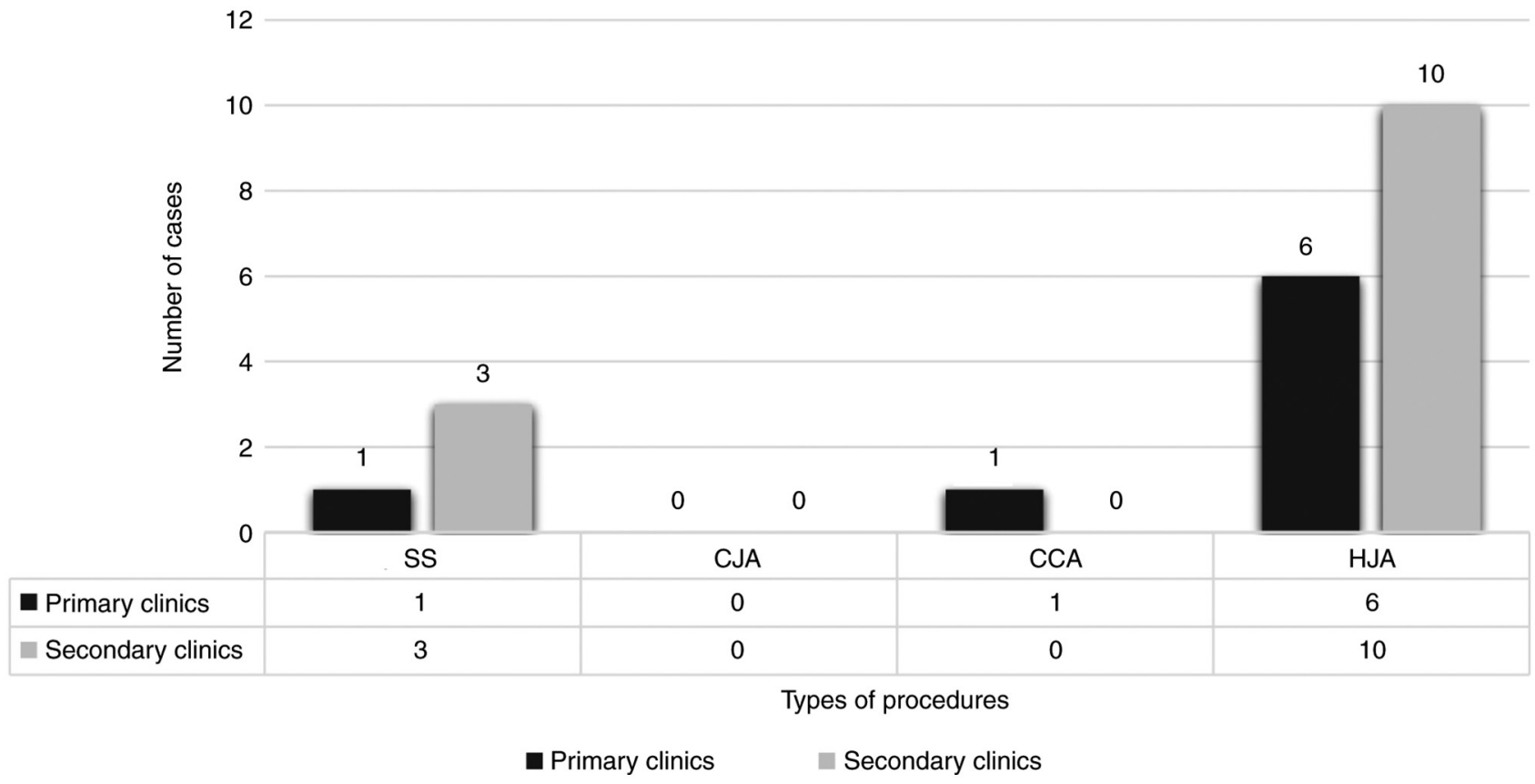

Figure 3. Types of surgical procedures that developed stenosis as a late-term complications, reported in conjunction with originating surgical centers. SS, simple suture; CJA, choledoco-jejunal anastomosis; CCA, choledoco-choledochal anastomosis; HJA, hepatico-jejunal anastomosis.

Analysis of the average length of the hospital stay showed a steady increase from 1.6 days for Group A to 8.8 days for Group B and to 11.8 days for Group C (Table II).

As we previously mentioned, the study considered both bile duct lesions and associated vascular lesions. Vascular lesions associated with LBDI, i.e., cases corresponding only to Group C, were recorded for 20 cases (19\% of the total). From a topographical viewpoint, these lesions were most often located at the level of the right hepatic artery (RHA), with 12 cases ( $60 \%$ of Group $\mathrm{C}$ and $12 \%$ of the total), followed by the common hepatic artery (CHA) with 3 cases $(15 \%$ of Group $\mathrm{C}$ and $3 \%$ of the total). The rarest vascular injuries were left hepatic artery (LHA) lesions with 1 case (5\% of Group C, $1 \%$ of the total) and 3 cases (15\% of Group C, $3 \%$ of the total) with portal vein $(\mathrm{PV})$ lesions.

\section{Discussion}

As the statistical analysis shows, of the 28 cases in which a primary repair was attempted at the same center where the lesion they was induced (the situation of secondary clinics), patients had a long-term progress worse than those operated on in centers with higher experience in hepato-biliary repair surgery (OR: 7.0, 95\% CI: 2.5-19.6; $\mathrm{P}<0.01$ ). General unfavorable progress is dictated by the induction of stenosis, as a highly unfavorable complication, especially of bilio-digestive anastomoses, but also of simple sutures performed as a means to solve laparoscopic bile duct injuries (LBDI). Regarding stenosis, as a specific complication, this claim is supported by the higher chance of stenosis (OR:7.0, 95\% CI; $\mathrm{P}=0.02$ ) for the group of patients operated per primam in secondary centers compared to those operated and treated in primary centers. By comparing the tendency of stenosis of the two subgroups of patients, a clear difference is clearly observed to the detriment of patients who underwent primary repair attempted by the same team that caused the iatrogenic lesion; among the 4 SS that caused late stenosis, 3 were carried out at primary clinics, respectively, and of the 16 hepato-jejunal anastomoses (HJA) which resulted in stenosis only 6 came from the two clinics experienced in hepato-biliary surgery. Although, in absolute numbers, the number of cases are smaller compared to wider international studies, the conclusions are similar and fully support this hypothesis, being consistent with publications by prestigious surgeons, such as studies of Stewart and Way (3) and Lillemoe et al (8).

The average length of hospitalization time increased, as expected, in proportion to the severity of the lesion; thus, cases in Group $\mathrm{C}$ required the longest period of admission, with an average of 11.8 days $(\mathrm{P}=0.001)$.

The statistical analysis of the costs recorded compared among the 3 lesion groups revealed a clear differentiation as well as an obvious growth trend directly proportional to the severity of the lesion $(\mathrm{P}<0.001)$. In this regard, comparing Group B with Group A had a P-value of 0.002 and comparing Group C with Group B also has a P-value of 0.002 .

The risk of requiring invasive surgery as a definitive method of treatment compared to the need for minimal-invasive approach also increased with advancement in the lesion groups $(\mathrm{P}<0.001)$. The difference between Group B compared to Group A was $27.7 \%$ (95\% CI: 5.5-138.9, $\mathrm{P}<0.001$ ), in favor of invasive interventions. However, if we translate this analysis between Group C compared to Group B (OR: 1.8, 95\% CI: 0.4-9.0; $\mathrm{P}=0.450$ ), no statistical significance was found. Therefore, the difference was no longer obvious between these classes, suggesting that only Group A required mainly minimally invasive interventions that no longer were useful in the context of complex injuries such as those found in Groups B and C.

This series of cases revealed a rate of 20\% LBDI associated with vascular injury, which is consistent with most published 
international studies (9). The consistency of this value leads us to believe that the seriousness of this possible complication is undervalued by most surgeons, especially in the context where laparoscopic cholecystectomy tends to be regarded as a trivial intervention with minimal risks to the patient $(9,10)$. The analysis of vascular lesions associated with ductal bile lesions reveals, predictably, that the most frequently injured in a traumatic event include RHA (in our study rated with $60 \%$ of lesions of LBDI with concomitant vascular interest, respectively $11 \%$ of the total LBDI in the study). Although the percentage itself is higher than that reported in the literature [12\% by authors such as Singh et al (11) and Deziel et al (12)], this study is relatively narrow in representation and includes cases that have also been submitted from less experienced centers, compared to the cited studies that enrolled top-rated reference surgical centers.

The statistical analysis allowed us also to note that the risk of vascular injury significantly increased the closer the LBDI was with the main biliary convergence $(\mathrm{P}=0.01)$. We expected that the vascular-associated lesions, interpreted as a whole, would lead to a severe increase in the incidence of late stenosis. However, this expectation was contradicted by the statistical analysis $(\mathrm{P}=0.4)$, which showed only a marginal increase in this risk. When isolating lesions on CHA from the entire group of vascular lesions, there was clearly an increased risk of stenosis of the procedures chosen to restore the hepatobiliary continuity. Furthermore, we can see that vascular lesions, being the main criterion for Group C classification, which, in turn, generated significantly higher LBDI case management costs, are therefore a significant risk factor that directly influences the socio-economic impact on these surgical cases.

The overall incidence rate of iatrogenic lesions recorded in this analysis was $0.65 \%$, a higher level than the $0.5 \%$ threshold cited in most international studies (13-15).

In conclusion, the present study confirms that the incidence ceiling of LBDI during laparoscopic cholecystectomy (LC) did not fall below the threshold of $0.5 \%$, which is double than the incidence rate reported by the most significant studies analyzing the incidence of open vs. laparoscopic incidence rates during LC (14). It is obvious that there is still much effort to be put into significantly lowering this threshold $(15,16)$. One way towards reaching this goal should be that all surgeons strictly adhere to the basic concept of always obtaining the critical view of safety advocated by Strasberg (17) and outlined by many studies published over the years $(18,19)$.

Following the analysis of surgical treatment options (both open and laparoscopic), factoring in the clinical results obtained (immediate and remote) for each method, we developed a guide with treatment options for the LBDI diagnosed during surgery or in the immediate postoperative period. The choices are presented according to levels: first intention, 2nd line, 3rd line, and so on.

Although our study has resorted to the creation of an internal classification system, it has minimal relevance to well-established main bile duct (MBD) injury reporting systems; thus, the recommendation guide will consider the most widely recognized Strasberg classification (Fig. 4).

Situations falling within Group A. For situations falling within Group A: i) If endsocopic retrograde cholagiopancreatography
(ERCP) is available, then it is preferable to perform it together with the endoscopic papilosphincerotomy (EPS) on a comfortable length followed by placement of a bile stent with appropriate characteristics (caliber, material, anchorage system). In this way the evolution is the closest to being considered ideal and the chances for favorable resolution are maximum. ii) If ERCP is not possible, than it is preferable to fully stabilize the case and subsequently to refer it, as soon as the general condition permits, to a clinic or center of excellence in hepato-biliary surgery where EPS with stenting can be performed. Stabilization measures should include placement of a peritoneal polyethylene drainage tube in the proximity juxta of biliary lesions (usually in subhepatic space), i.v. hydroelectrolytic and volemic support, nasogastric probe, antibiotic medication with tropism on the bile ducts, non-steroidal anti-inflammatory, antispastic and antalgic medication.

Situations falling within Groups $B$ and $C$. For situations falling within Groups B and C: i) If the clinic in which the surgical team that determined the LBDI also has the necessary experience in the repair of the hepato-biliary tree, then it is preferable that the case be taken over by that team. The advantages are, of course, multiple, primarily logistical in nature. The solutions for repair are in close connection with the type of injury as follows:

For Strasberg type D injuries. i) Primary option: If we have a type D lesion without associated vascular damage and the diameter of the defect is between 3 and $5 \mathrm{~mm}$, the first option included primary suture with an 4.0/5.0 absorbable monofilament surgical thread and close proximity drainage for monitoring. This solution can be performed laparoscopically (especially if the lesion was immediately identified intraoperatively) but also openly. ii) Secondary option: If the site of the ductal lesion has been deprived of vascular support (on a variable length) by a concomitant vascular impairment, then the primary suture is considered as prohibited because bile leaks and consecutive choleperitoneum will most likely be recorded at this level, starting with the very first week postoperatively. Thus, in these situations, an endoscopic radiological-guided stenting is indicated. iii) Third option: If the initial lesion has evolved from class D to class E, then open surgical reintervention is the highly recommended, the options available being those discussed in the section below.

\section{For Strasberg type E injuries}

If the main biliary convergence is free: i) Option 1: HJA should be carried out and has undeniable advantages, such as a good vascular inflow at the level of the bile duct, from the intestinal wall, but also a reduced mounting tension, due to the mobility of the jejunal loop, which can be properly ascended in such a way as not to endanger the anastomosis; bilio-digestive derivation with a free loop, in the absence of Oddian sphincter obstacles, is not subject to such a pressure regime, since the higher pressure gradient in the main bile duct and lower at the level of the loop will cause a completely free transanastomotic leakage of the bile. ii) Option 2: Hepatico-duodenal anasatomosis (HAD), which confers the advantage of an immediate proximity between the two 


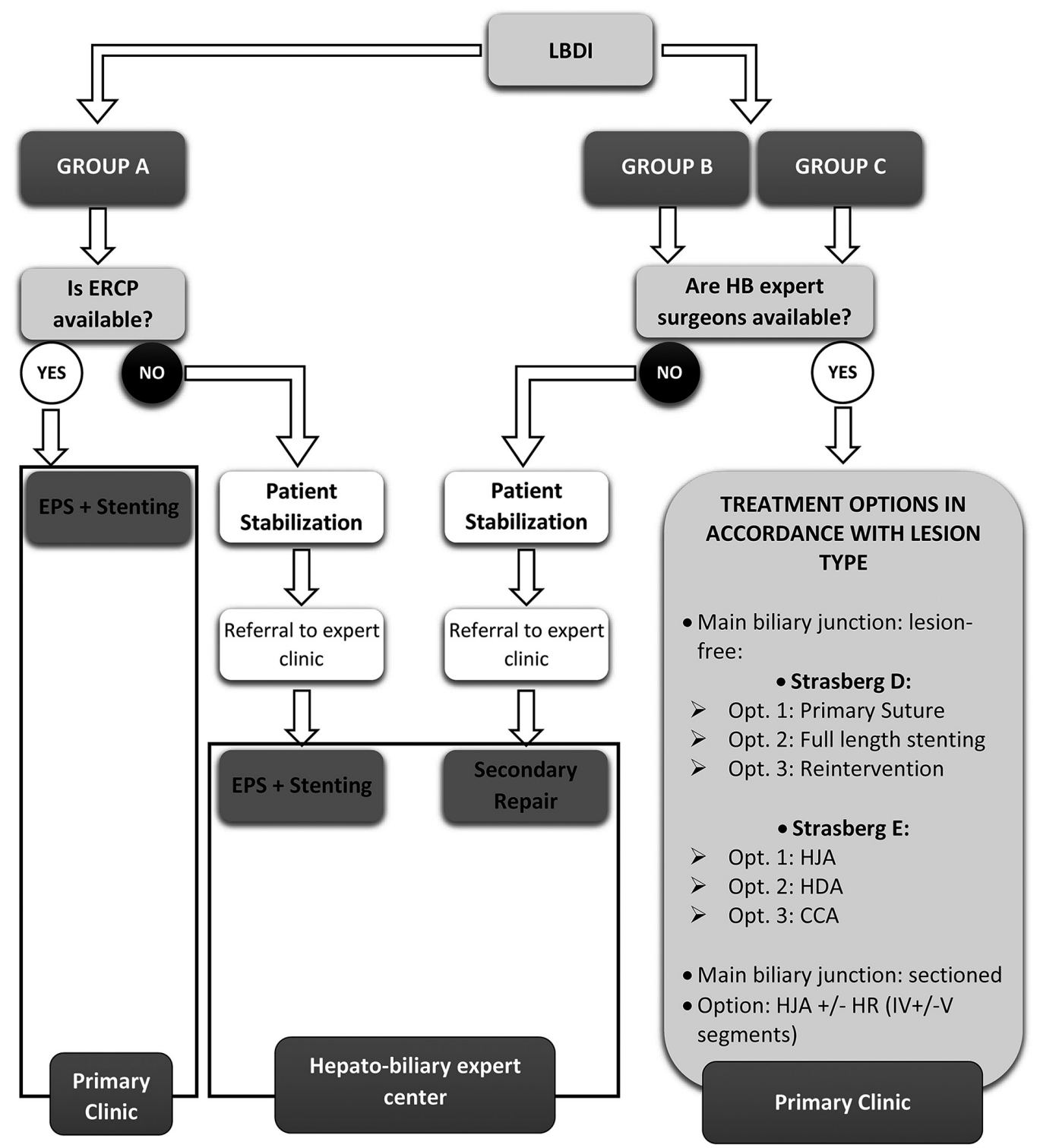

Figure 4. Complete layout of the proposed algorithm for managing clinical cases with recorded laparoscopic bile duct injuries (LBDI) during surgery or in the immediate postoperative stage. ERCP, endsocopic retrograde cholagiopancreatography; EPS, endoscopic papilo-sphicterotomy; HB, hepatobiliary; HJA, hepatico-jejunal anastomosis; HAD, hepatico-duodenal anasatomosis; CCA, choledoco-choledochal anastomosis.

structures as well as being less technically challenging. Other advantages include easy construction of the anastomosis of at least $1 \mathrm{~cm}$ in diameter; benefits for the duodenal wall in regards to good vascularization; installation of axial drainage with trans-ligamentary externalization and; fnally, remote revision of the anastomosis by means of upper digestive endoscopy (UDE), an advantage that no other bilio-digestive anastomosis has. Of course, the main disadvantage is the tendency of the duodenum to descend, especially during heavy food intake, exerting a higher tension in the anastomotic assembly and thus having an increased risk of fistulization, even if an extensive Kocher maneuver is performed for mobilizing the duodenal frame. In addition, duodenum anastomoses present a higher risk of biliary contamination, with all consecutive shortcomings: repeated episodes of cholangitis or even liver abscesses. iii) Option 3: Choledoco-choledochal anastomosis (CCA), if the following conditions are met: minimal distance between the two resulting biliary stumps, no tension whatsoever in the resulting final anastomosis, no associated vascular lesion is recorded, the use of monofilament surgical wires with fine round needle tips and with the knots executed outside the lumen. However, the inconveniences of this method of repair are multiple. The diameters of the two stumps are narrow with an opening of less than $10 \mathrm{~mm}$, thus making an end-to-end (E-E) anastomosis with separate stiches almost impossible. It is also difficult to assess the degree of desiccation of the bile ductal wall (even in the absence of a clear vascular lesion) induced by the electrosurgical unit during the LC. This method must therefore be viewed with caution. For the reasons exposed, many authors consider as mandatory the use of a prosthesis inside the T-T anastomosis with a trans-ligamentary externalized axial drainage, left in place for at least 6 months or the use of a biliary stent. These methods are intended to calibrate the anastomosis, thus preventing late stenosis, but contributing to the reduction of endoluminal pressure on stiches because, of all methods, the high endoluminal pressure 
regime in these situations is much higher than in any other type of bilio-digestive derivation.

If the main biliary convergence is affected. If the main biliary convergence is affected by the transection line of the LBDI, HJA is the only viable option, even when sometimes a partial liver resection (LR) of the IV and Vth segment must be practiced in order to gain access here for the jejunal loop. If the clinic in which LBDI was registered does not have an expert or group of surgeons experienced in this type of hepato-biliary repair surgery, then the recommendation is for complete stabilization of the case (under the same conditions as in the previous situation, with the difference that, this time, given the increased complexity of the ductal lesion with or without an associated vascular lesion, the patient's supportive measures are more laborious) and then transfer of the case to an expert center where it will be re-evaluated in order to choose the optimal treatment option.

The iatrogenic lesions of the main biliary pathways are far from being completely clarified and still represent a difficult surgical situation during open and laparoscopic surgeries. The outcome of these situations is intricately linked with the actual moment of discovery of the lesion and the surgical methods for repairing such defects and implies many options that an experienced surgeon must be aware of.

\section{Acknowledgements}

Not applicable.

\section{Funding}

No funding was received.

\section{Availability of data and materials}

The data sets that this study has been drafted upon are available from the corresponding author through E-mail request in a reasonable manner.

\section{Authors' contributions}

CM drafted the overall study guidelines. CM, DC, and FDG were responsible for designing the surgical strategy. GG and ER performed the statystical analyses. All authors read and approved the final manuscript for publication.

\section{Ethics approval and consent to participate}

Since this was a retrospective analysis, no ethical approval or consent was needed from the supporting institution or patients.

\section{Patient consent for publication}

Not applicable.

\section{Competing interests}

The authors declare they have no competing interests.

\section{References}

1. Reynolds W Jr: The first laparoscopic cholecystectomy. JSLS 5: 89-94, 2001.

2. Berci G: Laparoscopic cholecystectomy viewed from the USA. Aust N Z J Surg 61: 249-250, 1991.

3. Stewart L and Way LW: Bile duct injuries during laparoscopic cholecystectomy. Factors that influence the results of treatment. Arch Surg 130: 1123-1129, 1995.

4. Nuzzo G, Giuliante F, Giovannini I, Murazio M, D'Acapito F, Ardito F, Vellone M, Gauzolino R, Costamagna $\mathrm{G}$ and Di Stasi C: Advantages of multidisciplinary management of bile duct injuries occurring during cholecystectomy. Am J Surg 195: 763-769, 2008.

5. James RH, Brigstocke JR, Shields DA and Scurr JH: Medicolegal claims following laparoscopic cholecystectomy in the UK and Ireland. Ann R Coll Surg Engl 92: 286-291, 2010.

6. Grigoriu ME, Costea RV, Grigoriu CI and Furtunescu FL: Endoscopic management of choledocolithiasis related to periampullary duodenal diverticula. Med Surg J 122: 102-108, 2018.

7. Bismuth H: Postoperative strictures of the bile ducts. In: The Biliary Tract V. Blumgart LH (ed). Churchill-Livingstone, New York, NY, pp209-218, 1982.

8. Lillemoe KD, Martin SA, Cameron JL, Yeo CJ, Talamini MA, Kaushal S, Coleman J, Venbrux AC, Savader SJ, Osterman FA and Pitt HA: Major bile duct injuries during laparoscopic cholecystectomy. Follow-up after combined surgical and radiologic management. Ann Surg 225: 459-471, 1997.

9. Nuzzo G, Giuliante F, Giovannini I, Ardito F, D'Acapito F, Vellone M, Murazio M and Capelli G: Bile duct injury during laparoscopic cholecystectomy: Results of an Italian national survey on 56591 cholecystectomies. Arch Surg 140: 986-992, 2005.

10. Tzovaras G and Dervenis C: Vascular injuries in laparoscopic cholecystectomy: An underestimated problem. Dig Surg 23: 370-374, 2006.

11. Singh K, Singh R and Kaur M: Clinical reappraisal of vasculobiliary anatomy relevant to laparoscopic cholecystectomy. J Minim Access Surg 13: 273-279, 2017.

12. Deziel DJ, Millikan KW, Economou SG, Doolas A, Ko ST and Airan MC: Complications of laparoscopic cholecystectomy: A national survey of 4,292 hospitals and an analysis of 77,604 cases. Am J Surg 165: 9-14, 1993.

13. Lalisang TJM, Situmorang I, Ibrahim F, Widianto P and Marbun VMG: Management of post-cholecystectomy bile duct injuries without operative mortality at Jakarta tertiary hospital in Indonesia-a cross-sectional study. Ann Med Surg (Lond) 62: 211-215, 2021.

14. Jajja MR, Laboe A, Hashmi S, Nadeem SO, Sayed BA and Sarmiento JM: Standardizing diagnostic and surgical approach to management of bile duct injuries after cholecystectomy: Long-term outcomes of patients treated at a high-volume HPB center. J Gastrointest Surg: Feb 2, 2021 (Epub ahead of print).

15. Ray S, Sanyal S, Das S, Jana K, Das AK and Khamrui S: Outcomes of surgery for post-cholecystectomy bile duct injuries: An audit from a tertiary referral center. J Visc Surg 157: 3-11, 2020.

16. Barauskas G, Paškauskas S, Dambrauskas Z, Gulbinas A and Pundzius J: Referral pattern, management, and long-term results of laparoscopic bile duct injuries: A case series of 44 patients. Medicina (Kaunas) 48: 138-144, 2012.

17. Strasberg SM: Biliary injury in laparoscopic surgery: Part 1. Processes used in determination of standard of care in misidentification injuries. J Am Coll Surg 201: 598-603, 2005.

18. Sgaramella LI, Gurrado A, Pasculli A, de Angelis N, Memeo R, Prete FP, Berti S, Ceccarelli G, Rigamonti M, Badessi FGA, et al: The critical view of safety during laparoscopic cholecystectomy: Strasberg yes or no? An Italian multicentre study. Surg Endosc 35: 3698-3708, 2021.

19. Strasberg SM and Helton WS: An analytical review of vasculobiliary injury in laparoscopic and open cholecystectomy. HPB (Oxford) 13: 1-14, 2011. International (CC BY-NC-ND 4.0) License. 